\title{
Symmetry and Rhythm in the Art
}

\author{
Orazkulova Kaldigul \\ Mertenov Yeldos \\ Ryssymbetov Yerzhan
}

Kazakh National Academy of Arts named after T. Zhurgenov

Kaldigul.71@mail.ru

\section{Doi:10.5901/mjss.2015.v6n2s4p151}

\begin{abstract}
The article is devoted to the notion "archetype", "symmetry", "rhythm" and their correlation with the method of structural analysis of the graphic composition. Analyzing the artistic compositions, the authors consider the archetype as a structural-substantial invariant, and symmetry as the universal operational principle. Considering the artistic images, the authors state the concrete examples of compositional decisions. Visual appearance of an illustrated object is revealed in a spatial composition structure. Along with it, in fine arts iconic monuments are represented in concave shape of three-dimensional modality. In this very case, traditional semiotic coding of three-dimensional space is a particular conventional meaning with systematically denoted visual perception. While analyzing a picture the modality must be considered. Turning to the concept of symmetry, formed in modern art history, it is stated that symmetry-asymmetry aspect occurs in all systems, which characterize the universe: as in science, so as in art. The concept of symmetry is a reality and beauty, as well as a creator of harmony. Symmetric elements - rectangle, circle, S-shaped forms are the manifests of a primary tectonic principle: rhythmic repetitions of the spatial structure are conscious copies of spatio-temporal environment. They are universal forms, archetypes.
\end{abstract}

Keywords: Symmetry, rhythm, binary coordinates, visual perception, structural-constructive invariants.

The issue of symmetry and rhythm in arts has always attracted attention of the humanity's best minds: philosophers, artists, architects. Even nowadays, when it seems everything has been learned, this very matter hasn't lost its importance. Scientists of the world, based on the researches of Leonardo da Vinci, Favorskii V.A. and many others, continue the search of symmetry and rhythm consistent pattern in works of art and architecture.

Symmetry in the art is balance, harmony, correlation of the parties and integrity. In the fine arts the creation of form and composition is considered as the symmetric pair. In the world all creatures have the symmetry, and they all are divided along the centre by the axle. In nature all the things and phenomena are subjected to the law of symmetry. All have symmetric axle. All is in balance according to the principle of dynamic symmetry. The ball and square are symmetrical to axle. In the fine arts the space is closely connected with the symmetry. Building of a composition in the fine arts is designated by the horizontal line. The transfer of drawings from the life on the symmetric space in the fine arts is the basis of Gestalt psychology and this can explain the origin of complex images. So as V. Vlasov marks "in the theory of art the symmetry is considered as a universal principle of "regulating of the sense space". The symmetric system is an archetype with assistance of which the connection of geometry to the graphic surface with themes, subjects and iconographic tradition" (Vlasov V., 2008, p. 799). Thus, we can consider the universal archetype and symmetry as the separate scientific direction. In the system of binary coordinates symmetric relations are universal structural code. The binary system of coordinates in its turn is universal in the culture that presents novelty for the world science.

If to conduct even small digression into the history with purpose of understanding its essence, we will notice that certain binary principles are not simply conditional epistemological methods. All «dual» epistemology from antiquity to structuralism begins from ontology of culture. In the system of culture, concerning thought and language nature of whole binary is very important.

As the problem examined by us is related to the fine arts, we distance ourselves from the general cultural principles to the plane of visual-artistic principles. The structural-functional analysis of artistic-visual archetype constants is the purpose of the article. Therefore at the analysis of compositions of the works of art we'll decide on «s-type» and cross-like archetype forms. In symmetric compositions a central axis plays the leading role.

The following research objectives: 
- defining theoretically "symmetry" and "rhythm", using science literature analysis;

- summarizing the universal operating principle;

- studying art images within the framework of issue researches;

In order to achieve these goals the methods of structural analysis and empirical research are used, as well as the methodological background of cultural and art ideas of world scientific mind.

Research's practical meaning is that the analysis of "S-shaped" works of arts compositions, cruciform works of arts of archetype forms entails new meanings and images emergence in the world art evolution.

It is known that understanding of archetype in mythology, literature, folklore study, analytical psychology and cultural studies originates from the tradition of the Young's understanding formed long time ago yet. Archetype notions in the fine arts are linked to the iconography schemes or certain plot-vivid (symbolic) structures. At the same time, we can understand «archetype in the art» as the primary elements of visual forms. The basis of building the composition in the fine arts is lines. In history of fine arts the change of lines shows the change of styles. Consequently, line is not simple outlining on the surface; this is traces of world outlook searches. The philosophical roots of visible primary elements of the form show that they have certain similarity with notion of archetype.

Any graphic composition is perceived from the aspect of the two structural modalities: plane and space. In accordance with it, any structural analysis is directed on the exposure of features of conditionally designed image in three-dimensional space or two-dimensional image on a plane. The different circular, spiral-like, winding compositions actually are only the images carried on the surface of a picture. We don't examine the structure of a round surface as it is only in topographical area of art. In this case a plane in the art from the conceptual point of view acquires other semantic modality. The visual type of the depicted object is exposed in construction of the spatial composition. At the same time, in the fine arts the monuments of iconic type are represented in a concave kind in three-dimensional modality. The visual features of three-dimensional modality are not exposed here. In this case the traditional semiotic encoding of threedimensional space is specific conventional sense and its system-marked visual perception is given here. Thus, an inherent feature and semantic conventional connection of the compositional elements located in space are formed. At the analysis of picture, at observation of the aspect from the external or internal side, it is necessary to take into account both modalities.

The plane modality of the compositional plane perception, i.e. the review from this perspective is not threedimensional space review; these are the cultural-semantic layers of the unconscious formed during ages. These principles arose up as a result of recoding the plane of syncretism unity semantic block. The culture tries to hide the structural bases lying deep from a subject. Nevertheless, the distances in time do not deprive them and, vice versa, approach them to the most adequate interpretation. The principle of structural order of the image located on twodimensional plane in stable cultural tradition is not only individual, experience, but collective too. The masters of antiquity brought it on the incredibly high level from the aspect of function and method of the art. In strong syncretism-intuitional senses, the continuous connection with the past is kept. However, visually encoded senses, depicted on two-dimensional plane, are developed in the culture in accordance to its regularity secretly from the subject.

The development of compositional forms is considered more from position of structural-logical scheme, than historiography one. The central-symmetric composition formed in deep antiquity is met in the art today.

If to stop on the notion of symmetry formed in the modern study of art, it should be noted that the aspect of symmetry-asymmetry is met in all systems characterizing universe both in science and the art. The notion of symmetry is reality and beauty, at the same time, creator of harmony.

Exploring the creation of form and laws of perception from the aspect of methodology of Gestalt school, Rudolf Arnheim pays a lot of attention to symmetry. «The development of graphic form takes place on the basis of the nervous system characteristic properties, which only insignificantly is subjected to the effect of cultural and individual differences. Namely because of this reason the child's pictures in the whole world are essentially similar to each other, and in the works of arts of the early history epochs of different civilizations has striking likeness» (Arnheim R., 2012, p. 392).

Modern humanitarian thought extended the value of the symmetry notion. If the empiric observation of symmetric image prevails in the traditional study of art, in the works of P. Florenskii, E. Husserl, A.F. Loseva, E. Panovskii, Yu. Lotman the opinion was formed that symmetry is an ideal principle of semantic space arrangement. Yu. Lotman marks the following: «... the spatial organization is one of the universal facilities of construction of any cultural models» (Lotman Yu., 2000, p. 704). Symmetry links the real life to the perishable world parallel to it.

Thus, the archetype of symmetry proves the invariance of cosmograms, symbols and graphic images. For example, patterns and ornaments are carried out according to the laws of symmetry. R. Arnheim writes the following: «The development of graphic form takes place on the basis of the nervous system characteristic properties, which only insignificantly is subjected to the effect of cultural and individual differences». 
A. Pelipenko expressed the thought that « a number row can be grouped as follows: from one unit to three ones the most sense-forming energy and semantic valence. Further, from four to seven and from seven to ten. Next, from ten to thirteen and etc. All further values, approximately from middle of the second tens, in any case, are perceived as derivatives, and first of all mathematically (instead of universally) functional» (Pelipenko A.A., 1998, p. 376). First "1", "2", "3" are the universal and comprehensive potential of number three, which modulates the three-dial principle. The triple system forms the invariant symmetric module.

A visual code on any plane is a point. "The simplest and at the same time universal and ideal geometric symbol is the point... The point is a symbol of absolute. The absolute is an eternally unchanged fundamental principle of all existing things". Serikbol Kondybai writes that "a round sign is a point in a circle, i.e. it consists of two elements - a circle and point. A point is the sign of the Most high, and a circle is the symbol of Universe» (Kondybai Serikbol, 2004, p. 504). Thus, he implies that through this sign all exiting things on earth are understood - the ray of light is "opening" and "reflection". "The central point is invisible" here, and rays outgoing from this point are "visible". "The centre and periphery" comes from here. These two oppositions have concrete dualistic forms.

Correlation of point and "1" generates the dual relation: point and environment. Continuation of the point generates a line. A point (centre) is clean sense and archetype abstract nature. Horizontal and vertical lines formed from a point possess sacral sense. The right and left, low and top means spatial/temporal empiric time. A diagonal line between horizontal and vertical lines means motion. The angle of diagonal line appeared later, because it is located awry in relation to the horizontal and vertical lines.

Horizontal and vertical lines on the plane, located in opposition, create two primary elements, and primary tectonic forms - a quadrangle and cross.

A cross is the synthesized and finished form. Quadrangle is reduction form. The quadrangle, created owing to the connection of horizontal and vertical lines, models an oppositional visual code: sacral/profane. A quadrangular structural element surrounds a point and is the centre of horizontal or vertical turn of the point form. The primary element of improved and finished quadrangular form is the right quadrangle. The right quadrangle in any context implies stability, firmness and equilibrium. The right quadrangles were the basis of architectural buildings, and the compositional structure of pictures was also based on this form.

The right quadrangle is similar to any reserved form located on a surface. It takes place as a result of limitation of the internal and external space. So, four lines of form serve as limiters of the symmetric area.

Also the wide distributed figures are square and circle. A circle appeared before all the other forms. How Pelipenko A.A. and Yakovlenko I.G. state: "A circle is the result of primary mental impulse, unwinding the point on a plane" (Pelipenko A.A., 1998, p. 376), and continue further: "The inertness of a round model is connected with the fact that having unlimited number of symmetry axles, a circle is indifferent and inert in relation to any transformations on a frontal plane, but extraordinarily vulnerable in relation to any transformations in the space" (Pelipenko A.A., 1998, p. 376).

Serikbol Kondybai named a geometrical figure consisting of the intersecting lines on one point - shogym tanba. It consists of one cross point and a few (two, four, six, eight, twelve and etc.) lines. A figure can be represented in such kind and also located in a circle. This sign has two fundamental elements, first - point, second - a line of ray and a line of circle (we perceive a ray and circle as interchangeable elements). So, these two elements make oppositional pairs to each other: Point and ray/circle or Centre and periphery. If to count up that the stated figures are represented not in twodimensional (width, length), and in three-dimensional space (width, length, height or depth), then we would see them in the form of the inverted cone...

Thus, the main innovation of transformation of this form lies in the "appearance of opposition of "up-down" (Kondybai Serikbol, 2004, p. 504). The round form of "shogym tanba", and invariant figures explain the deep content of a myth, such notions as four sides of the world, three worlds. The structure of this world became a constant form and compositional basis for artistic works. It should be noted that such forms are met in the art of all peoples of the world.

Kazakh nomad's tent is also the specific mini copy of the universe. B.K. Baizhigitov deeply analyzed the form of nomad's tent, its artistic features and functions. A philosopher-art critic writes the following about construction of a nomad's tent: "The external form of a nomad's tent is made according to the natural laws. A round and spherical structure is very steady to high winds and other kinds of natural phenomena", then he further continues: "form of a nomad's tent and shanyrak (shanyrak - a structural element crowning the dome of a nomad's tent in the form of the latticed crosspiece, signed in the circle - author's note) in the form of circle, and also the latticed cross-piece represents "mini model of space" (Baizhigitov B.K., 1998, p. 192), thus, marks the harmony of nomad's tent with nature".

Also the S-like figure is wide-spread. This figure does not look like the reserved circle; it is antipode to the circle, as it reproduces the idea of the open space. S-like figures, looking like a triangle, mean the universal triune rhythmicdynamic module. S-like figures consist of three elements, first - primary one, second - finishing one, and third - uniting 
one.

S-like figures look like a circle by that at both these figures a circulating point begins from a centre. Ornamental composition of Saxon epoch is based on this principle.

The rhythm of ornament on a plane is repeated both on a horizontal and vertical line. A horizontal line divides a top and bottom, as it can be also examined as a border between the past and future. The Kazakh outlook composition of triplicity - lower, middle and upper is the sense understanding of the world. At all peoples of the world a horizontal line implies stability, and a vertical line is growth, prosperity or explorer of two worlds.

In the Kazakh art, S-like images of animals are often met, which were inherited from Saxon people. "An animal is in the boundary state - between life and death. Therefore, the lower part of a body, symbolizing its physical essence, is turned inside out on 180 degrees in relation to the axle, as a result a body loses the support in a physical world, i.e. it stops to feel earthly ground under the feet, while the upper part of a body, symbolizing its spiritual essence (soul), is still in the real world and still fight for the existence" (Butkevich L.M., 2005, p. 267).

We see that what from the first view is a very simple composition structure possesses internal energy and dynamic force based on a rhythm. The doctor of the philosophical sciences Alibek Kazhgaliuly thus marks the role of rhythm in the art: "... ornament appeared simultaneously with the image, but not from the image. It is not necessary to search an ornament in the image - it is absent there, as ornament appears between images. The ornament is connection, arising between its objects, and rhythm is a basis for appearance of this connection" (Kazhgaliuly A., 2003, p. 456).

"The rhythm in ornament indeed displays itself as fastening sense content beginning, promoting to transformation of the sense picture in the artistic image. It formalizes ornament - creates the certain conserving form, sweeping aside the unimportant and keeping the unique one, i.e. a rhythm fastens, and takes off the ornament content in the dialectical sense, translating the mythological content in the artistic image" (Butkevich L.M., 2005, p. 267).

At the analysis of art works the question can arise from where the rhythmic repetition of one motif appeared, i.e. so called "repeat nature". M. S. Kagan on this occasion marks that it is "explained, of course, not by the abstract aesthetic pleasure, which a man gets from a rhythm... In fact, in canvas, on the walls of cylindrical vessel, walls of building, rectangular carpet, and the round dish - in all these objects the decorated surface flows infinitely..." (Kagan M.S., 2008, p. 408).

Thus, as the result of the structural-functional analysis of art-visual archetype constants we have come to the following conclusions. Transferring sketches from life into symmetrical space in fine arts is the foundation of Gestalt psychology, and that is why it can explain the emergence of complex images. In the art theory symmetry is considered as the universal principle for ordering sense of space. In this case symmetric system is the universal archetype that can be as novelty and progress perspective in the future world science apart from scientific direction. Primary visual form elements are the archetypes in art. The principle of image structural order, located at the two-dimensional plane, in the established cultural tradition, is not only one individual experience, but also the collective. Symmetry connects present life to its parallel mortal world. Symmetry's archetype proves the cosmogram, symbols and graphic images invariance. The first "1", "2", "3" - are the universal and overall potential of the number three that models the triple principle. The triple system creates invariant symmetrical module. Based on that, visual code at every plane is a dot, which is an absolute symbol, when a dot in circle is the sign of Almighty. Circle is the symbol of the Universe. The ratio between a dot and "1" emerges dual relation: the dot and the environment. The dot continuation emerges line. The dot (center) is a pure sense and archetypal abstraction. Horizontal and vertical lines, formed from the dot, have a sacral meaning. Shogym tanba has also a great meaning, explaining deep content of a myth, such notions as four cardinals, three worlds. This world structure has become a constant form and compositional foundation for works of art. Note that such forms occur in arts of all peoples in the world. From this point of view an example is the yurt of the Kazakhs, which is a peculiar mini copy of the Universe, expressed by means of shanyrak. Triplicity composition of the Kazakhs worldview is the lower, medium and upper are the notional understanding of the world. In the Kazakh art you can often meet S-shaped images of animals that were inherited form the Saka. Important role of the rhythm in art is followed in the Kazakh ornament.

As a result of empiric research performed by the authors of this paper, in which took part 120 students of the art history faculty of the T. Zhurgenov, Kazakh National Art Academy (Almaty city, Kazakhstan) were compared survey results in absolute and relative data, has been established a clear pattern between numerical series of S-shaped figurative figures in the Kazakh ornament that proves the existence of the archetype.

The higher symmetric elements considered are a quadrangle, a circle, while the S-like forms are manifests of the primary tectonic principle: rhythmic repetitions of the spatial structure are the conscious copies of spatial-temporal environment. These are universal forms, and archetypes lying in the deep sub-consciousness. 


\section{References}

Arnheim R. (2012). The art and visual perception / Arnheim R. Transl. from Engl. Moscow, "Arkhitektura-S".

Baizhigitov B.K. (1998). Beineleu onerinin filosofiyalyk meseleleri: kenistik pen yakyt yrgagyndagy turakty suret yulgilery. Almaty: Falymolke.

Bessarabova E.B. (2014). Analysis of factors that influence the formation of impression about perceptive object. Universum: Philology and art history: electron, science magazine. № 8 (10). URL: http://7universum.com/ru/philology/archive/item/1534 (appeal date: 16.03.2015).

Boiko O.V.(2014). The art of tapestry is the most important component in forming interior art image. Universum: Philology and art history: electron, science magazine. № 10 (12). URL: http://7universum.com/en/philology/archive/item/1660 (дата обращения: 20.03.2015).

Butkevich L.M. (2005). History of Ornament. Humanitar. Publish. Center VLASDOS.

Kagan M.S. (2008).Selected works in 7 volumes. Volume V. Problem of theoretical art study and aesthetics. Saint-Petersburg: PH "Petropolis".

Kazhgaliuly A. (2003).Ornament organon. Almaty.

Kondybai Serikbol (2004). Argykazak mifologiasy. Birinshi kitap. Almaty: Dyke- Press.

Koshkina O.J. (2014).Plastic metaphor as the foundation of graphic works I.T. Bogdesco // Universum: Philology and art history: electron, science magazine 2014. № 8 (10) . URL: http://7universum.com/ru/philology/archive/item/1532 (appeal date 21.03.2015).

Kuznetsova-Bondarenko E.S. (2014). Structural analysis of stained-glasses in Holy Trinity Cathedral Simferopol city. Universum: Philology and art history: electron, science magazine. № 11 (13)

Lotman Yu. M. (2000), About the art. Saint-Petersburg: "Iskusstvo.

Mircea Eliade. (2000). Myths, Dreams and Mysteries. Harper \& Row, Pudlishers, New York Grand Rapids, Philadelphia, St. Louis, San Francisco, London, Singapore, Sidney, Tokyo, Toronto.

Muter R. (2009). General History of life. Moscow: Eskmo.

Pelipenko A.A., Yakovlenko I.G. (1998), Culture as a sytem. Moscow: "Yazyki russkoi kultury".

Philosophical Encyclopedic Dictionary. (2000). Moscow: INFA.

Veis G. (2007). General history of world culture. Moscow: Eksmo.

Vlasov V. (2008). New encyclopaedic dictionary of the fine arts: in 10 volumes. V. -VIII. Saint-Petersburg: Azbuka-klassik.

Wowk Anatole. (2006). English-Ukrainian Dictionary of Color Names and Color Science. New York. Paris. Sydney. Toronto.

Zuban A.N. (2014). Historical and stylistic analysis of the outdoor advertisement development in Russia. Universum: Philology and art history: electron, science magazine. № 10 (12) . URL: http://7universum.com/en/philology/archive/item/1659 (appeal date: 26.03.2015). 
